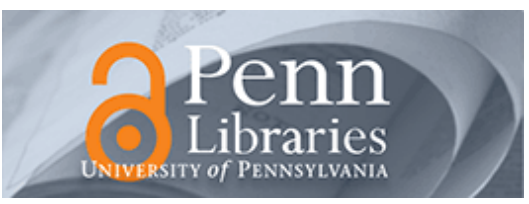

University of Pennsylvania

ScholarlyCommons

June 2007

\title{
The Measured Electric Field Spatial Distribution within a Metamaterial Sub-Wavelength Cavity Resonator
}

Thomas Hand

Duke University

Steven A. Cummer

Duke University

Nader Engheta

University of Pennsylvania, engheta@ee.upenn.edu

Follow this and additional works at: https://repository.upenn.edu/ese_papers

\section{Recommended Citation}

Thomas Hand, Steven A. Cummer, and Nader Engheta, "The Measured Electric Field Spatial Distribution within a Metamaterial Sub-Wavelength Cavity Resonator", . June 2007.

Copyright 2007 IEEE. Reprinted from IEEE Transactions on Antennas and Propagation, Volume 55, Issue 6, June 2007, pages 1781-1788.

This material is posted here with permission of the IEEE. Such permission of the IEEE does not in any way imply IEEE endorsement of any of the University of Pennsylvania's products or services. Internal or personal use of this material is permitted. However, permission to reprint/republish this material for advertising or promotional purposes or for creating new collective works for resale or redistribution must be obtained from the IEEE by writing to pubs-permissions@ieee.org. By choosing to view this document, you agree to all provisions of the copyright laws protecting it.

This paper is posted at ScholarlyCommons. https://repository.upenn.edu/ese_papers/285

For more information, please contact repository@pobox.upenn.edu. 


\title{
The Measured Electric Field Spatial Distribution within a Metamaterial Sub- Wavelength Cavity Resonator
}

\author{
Abstract \\ Through experimental investigation, a thin subwavelength cavity resonator was physically realized using a \\ bilayer structure composed of air and a negative permeability metamaterial structure one unit cell in \\ thickness. We designed and built the metamaterial slab with periodic metallic ring structures and \\ measured the spatial electric field magnitude in a cavity formed from this slab and a region of air, \\ showing that a subwavelength cavity can be realized. The measured electric field magnitude distribution \\ in the cavity matched very well with effective medium theory, showing that even a slab one unit cell in \\ thickness may be effectively equivalent to a thin homogeneous medium as far as the construction of a \\ sub-wavelength cavity is concerned, provided that the unit cell size is significantly smaller than the free \\ space wavelength.
}

\section{Keywords}

cavity resonator, metamaterials, microwave cavities, negative index of refraction, negative permeability

\section{Comments}

Copyright 2007 IEEE. Reprinted from IEEE Transactions on Antennas and Propagation, Volume 55, Issue 6, June 2007, pages 1781-1788.

This material is posted here with permission of the IEEE. Such permission of the IEEE does not in any way imply IEEE endorsement of any of the University of Pennsylvania's products or services. Internal or personal use of this material is permitted. However, permission to reprint/republish this material for advertising or promotional purposes or for creating new collective works for resale or redistribution must be obtained from the IEEE by writing to pubs-permissions@ieee.org. By choosing to view this document, you agree to all provisions of the copyright laws protecting it. 


\section{The Measured Electric Field Spatial Distribution Within A Metamaterial Subwavelength Cavity Resonator}

Thomas Hand, Student Member, IEEE, Steven A. Cummer, Senior Member, IEEE, and Nader Engheta, Fellow, IEEE

\begin{abstract}
Through experimental investigation, a thin subwavelength cavity resonator was physically realized using a bilayer structure composed of air and a negative permeability metamaterial structure one unit cell in thickness. We designed and built the metamaterial slab with periodic metallic ring structures and measured the spatial electric field magnitude in a cavity formed from this slab and a region of air, showing that a subwavelength cavity can be realized. The measured electric field magnitude distribution in the cavity matched very well with effective medium theory, showing that even a slab one unit cell in thickness may be effectively equivalent to a thin homogeneous medium as far as the construction of a sub-wavelength cavity is concerned, provided that the unit cell size is significantly smaller than the free space wavelength.
\end{abstract}

Index Terms-Cavity resonator, metamaterials, microwave cavities, negative index of refraction, negative permeability.

\section{INTRODUCTION}

$\mathbf{E}$ VER SINCE negative index materials were first studied [1], ways to utilize these materials in practical applications have been the focus of many research groups. It has been shown that a subwavelength cavity resonator can be envisioned using a bilayer structure involving a negative index metamaterial [2]. The wave vector inside a negative index material (NIM) slab is less than zero which thus allows it to advance the phase of the electric field by an amount proportional to its thickness. When a slab of right handed material is placed next to the NIM, the phase delay introduced by the positive index material (PIM) is compensated for by the NIM. Thus, the NIM acts to "undo" the phase delay accrued by the right handed material, allowing for a net phase shift of zero across this bilayer composite structure, which satisfies the condition for resonance. It was shown in [2] that when the slab thicknesses are chosen properly, the net phase delay accumulated across the bilayer structure can be equal to zero. This effect relies on a specific dispersion relation that requires one of the slabs to have a negative permeability. Conventional cavity resonators are made to be compact by loading them with a high dielectric constant material which reduces their

Manuscript received October 10, 2006; revised February 26, 2007.

T. Hand and S. A. Cummer are with the Department of Electrical and Computer Engineering, Duke University, Durham, NC 27708-USA (e-mail: thh5@duke.edu; cummer@ee.duke.edu).

N. Engheta is with the Department of Electrical and Systems Engineering, University of Pennsylvania, Philadelphia, PA 19104 USA (e-mail: engheta@ee. upenn.edu).

Digital Object Identifier 10.1109/TAP.2007.898630

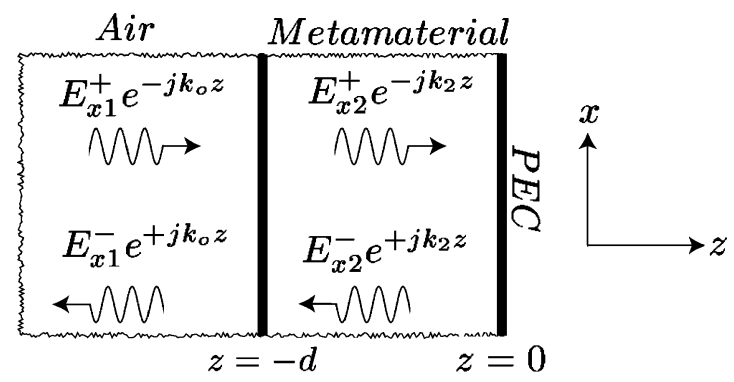

Fig. 1. Schematic of the fields inside the cavity, showing the incident and reflected waves in both slabs.

effective wavelength, and common techniques for this can be found in [3]-[5]. In contrast, this metamaterial approach can shrink the electrical size of the cavity smaller than half a wavelength.

Li, et al.[6] reported an experimental validation of a NIMbased compact cavity resonator. In their study, the resonator effect was observed by showing that the phase variation across the air/metamaterial structure was close to zero. This showed that the metamaterial slab advances the phase of the electric field to compensate for the phase lag through the PIM slab. It was also shown experimentally that the ratio of the slabs $d_{1} / d_{2}$ controls the resonance and not their sum $d_{1}+d_{2}$, as was originally envisioned in [2]. In our approach here, we aim to measure the amplitude distribution inside the cavity as detailed in [2] to reveal the basic physics of this class of resonator. The field magnitude spatial distribution inside the thin bilayer cavity should be approximately triangular in shape because of the discontinuous first derivatives of the electric field at the air/metamaterial boundary, which is due to change in sign in the effective permeabilities of the two slabs [2]. An experimental study that shows the effective discontinuity of the derivative of the electric field amplitude across a NIM/PIM interface is found in [7] and it is this physical effect that we will take advantage of for the realization of the compact cavity resonator. We show theoretically and experimentally that this class of subwavelength cavity resonator can be realized with a negative- $\mu$ only material as explored in [6], [8], [9].

\section{ANALYTICAL FORMULATION}

The analysis begins by solving for the electromagnetic fields inside a region loaded with two dielectric media with properties $\epsilon_{o}, \mu_{o}$ and $\epsilon_{2}, \mu_{2}$, as seen in Fig. 1. Throughout this paper, we suppress $e^{+j \omega t}$ time convention. We aim to show that a null in the electric field very close to the bilayer interface can be 
achieved that is significantly less than the $\lambda_{0} / 2$ required for conventional cavities. We will also show that the permittivity $\epsilon_{2}$ can be effectively neglected when a certain condition is met. An $\hat{x}$-polarized incident plane wave impinges upon the metamaterial slab of thickness $d$. A perfect electric conducting (PEC) plate is located at $z=0$ (Fig. 1), which acts to form the right side of the cavity.

The electric fields in both regions are expressed in terms of $+z$ and $-z$ traveling waves as

$$
\begin{aligned}
& E_{x 1}=E_{x 1}^{+}\left(e^{-j k_{o}(z+d)}+\Gamma_{1} e^{j k_{o}(z+d)}\right) \\
& E_{x 2}=E_{x 2}^{+}\left(e^{-j k_{2} z}-e^{j k_{2} z}\right) .
\end{aligned}
$$

Where $k_{o}=\omega / c$ is the free-space wave number and $k_{2}=$ $(\omega / c) \sqrt{\mu_{r 2} \epsilon_{r 2}}$ is the wave number of the metamaterial slab. Upon casting the magnetic field in terms of the electric fields given by (1) and (2) using $\mathbf{H}=(\nabla \times \mathbf{E}) /-j \omega \mu$, we solve for the reflection coefficient at the air/slab interface

$$
\Gamma_{1}=1-\frac{2}{1+j \frac{k_{o} \mu_{2}}{k_{2} \mu_{o}} \tan \left(k_{2} d\right)}
$$

Thus, the electric field distribution in the air is given by

$$
E_{x 1}=E_{o}\left[e^{-j k_{o}(z+d)}+\left(1-\frac{2}{1+j \frac{k_{o} \mu_{2}}{k_{2} \mu_{o}} \tan \left(k_{2} d\right)}\right) e^{j k_{o}(z+d)}\right] .
$$

A one dimensional resonant cavity would be formed by placing a PEC plane at any locations where $\left|E_{x 1}\right|=0$. Normally this requires PEC plane separation of at least $\lambda_{0} / 2$, but there are circumstances where this separation can be made shorter. If $k_{2} d \ll 1$, then $\tan \left(k_{2} d\right) \approx k_{2} d$, and if $k_{o}(z+d) \ll 1$, then $e^{j k_{o}(z+d)} \approx 1+j k_{o}(z+d)$. With these small argument approximations in which both layers are electrically thin, the field in the air medium $(z<-d)$ is given by

$$
E_{x 1}=2 E_{o}\left[1-\left(\frac{1+j k_{o}(z+d)}{1+j k_{o} \mu_{r 2} d}\right)\right] .
$$

It is evident that for $z<-d$, the only way for there to be a zero of $\left|E_{x 1}\right|$ close to the interface is when $\mu_{r 2}<0$. Also note that there is no $\epsilon_{r 2}$-dependence on the electric field when $k_{2} d$ and $k_{o}(z+d)$ are both small. If $\mu_{r 2}<0$, then the free space fields will be essentially the same if the fields are propagating $\left(\epsilon_{r 2}<0\right)$ or evanescent $\left(\epsilon_{r 2}>0\right)$ in the metamaterial slab. In either case, a null in $\left|E_{x 1}\right|$ will occur at $z=d\left(\mu_{r 2}-1\right)$, which means that distance between field nulls (effectively the cavity) can be made arbitrarily small if $\mu_{r 2}<0$ [2]. Fig. 2 demonstrates this through calculations of theoretical electric field magnitude versus position for various slab permeabilities using (4) at a frequency of $2.5 \mathrm{GHz}$, a metamaterial slab thickness $d$ of $15 \mathrm{~mm}$, and permittivity $\epsilon_{r 2}=1$. The air/slab interface is located at $z=-0.015 \mathrm{~m}$. It can be seen that the null position is strongly dependent on the permeability of the slab, for as $\mu_{r 2}$ increases from -2 to -0.5 , the null position pushes back closer to the

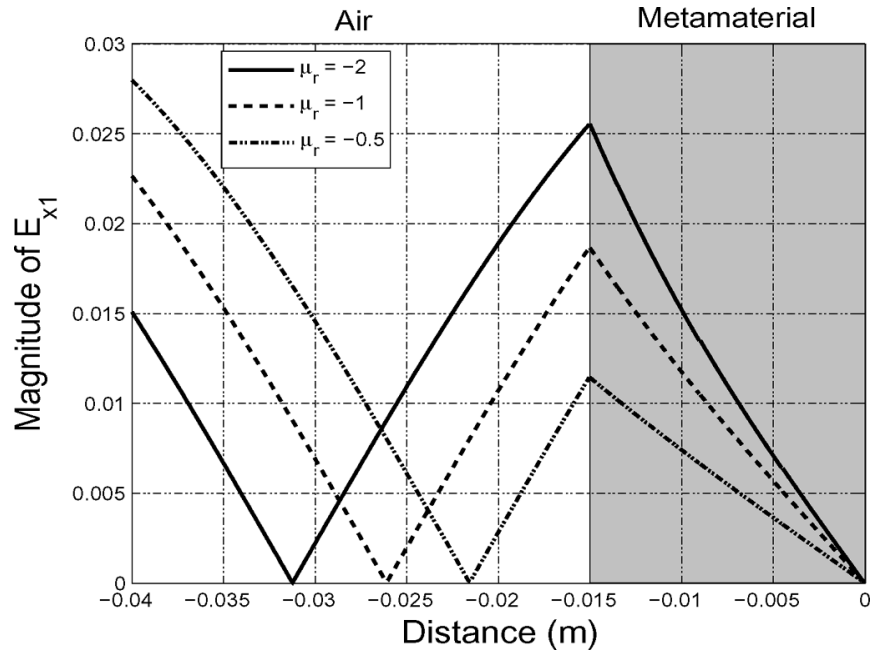

Fig. 2. Showing that small fluctuations in the slab permeability will cause noticeable variations in the electric field distribution for small $k_{2} d$. The plot was created for $f=2.5 \mathrm{GHz}$ and a metamaterial slab thickness of $1 \mathrm{~mm}\left(k_{2} d<\right.$ $0.03)$.

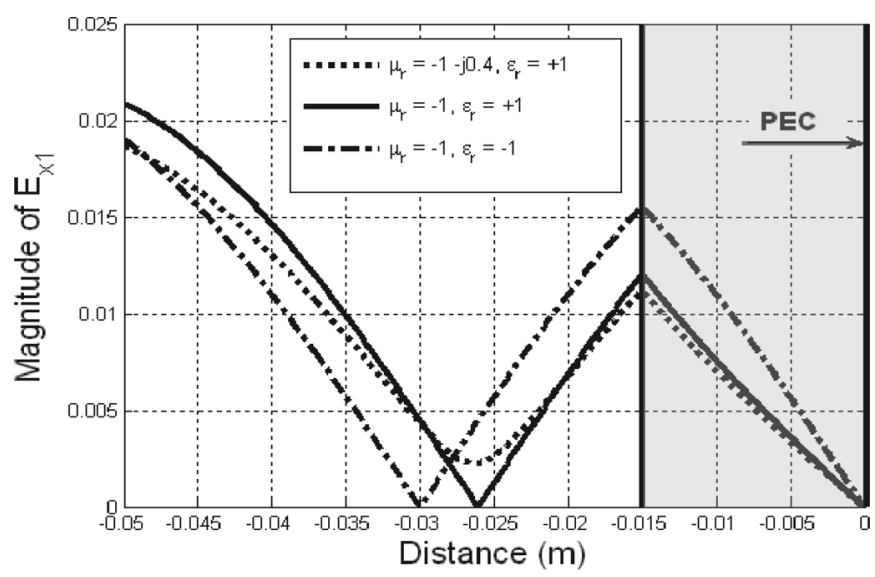

Fig. 3. Plotting the electric field structure for different material parameters at $2.5 \mathrm{GHz}$. Variations in the slab permittivity do not alter the electric field structure significantly, and this allows us to fabricate the compact cavity resonator using a negative- $\mu$ metamaterial. The cavity can still be formed even if the fields are evanescent in the metamaterial slab. It is also seen how magnetic losses prevent the electric field from reaching a null in the air portion of the cavity.

interface at $z=-0.015 \mathrm{~m}$. This clearly tells us that the subwavelength cavity can be formed with a negative- $\mu$ metamaterial, even if $\epsilon_{r 2}>0$, which will substantially simplify fabrication of such a resonator.

To confirm the weak effect the slab permittivity has on the spatial electric field structure, using (4), Fig. 3 shows $\left|E_{x 1}\right|$ for $\epsilon_{r 2}= \pm 1$ with $\mu_{r 2}$ fixed at -1 for $f=2.5 \mathrm{GHz}$ and $d=15 \mathrm{~mm}$. It is clear that this change in $\epsilon_{r 2}$ does not significantly affect the spatial field structure. In fact, the distance between nulls is slightly smaller when $\epsilon_{r 2}=1$, and thus a cavity could be made even smaller with such a bilayer structure. This is exactly the theoretical field distribution of [2], and is what we aim to fabricate and measure in our experiment. When the real part of $\epsilon_{r 2}$ and $\mu_{r 2}$ have different signs, no propagation can occur inside the metamaterial slab since $k_{2}$ is imaginary, which 
leads to an exponentially damped field in the slab. In Fig. 3, we observe that there is no propagation in the slab when $\epsilon_{r 2}=+1$ and $\mu_{r 2}=-1$ since the refractive index $n=\sqrt{\mu_{r} \epsilon_{r}}$ is imaginary, but the compact cavity can still be formed. This is because the requirement of continuity of tangential components of the magnetic field intensity given by

$$
\left.\frac{1}{-j \omega \mu_{1}} \frac{\partial E_{x 1}}{\partial z}\right|_{z=-d}=\left.\frac{1}{-j \omega \mu_{2}} \frac{\partial E_{x 2}}{\partial z}\right|_{z=-d}
$$

implies that

$$
\left.\frac{\partial E_{x 1}}{\partial z}\right|_{z=-d} \neq\left.\frac{\partial E_{x 2}}{\partial z}\right|_{z=-d}
$$

So the boundary conditions will support a discontinuity in the derivative of $E_{x}$ [2]. Because the derivative of $E_{x}$ is forced to change signs from the metamaterial slab to the air region, it quickly drops off immediately beyond the interface as can be seen in Figs. 2 and 3. This physical effect allows the shrinking of the cavity, and cannot be achievable with right handed materials because the gradient of $E_{x}$ would not be able to change signs. It is also important to note the effect that losses in the metamaterial slab have on the field structure in the cavity. Fig. 3 shows the electric field structure in the cavity for several values of permittivity and permeability. It is seen that the electric field cannot reach a null when magnetic losses are added. This is because part of the energy of the incident wave is absorbed by the metamaterial slab (mainly due to ohmic losses of the copper in practice), and the reflected wave amplitude is smaller than the incident wave amplitude, preventing a perfect standing wave pattern from forming in the air region as in the lossless case.

\section{Design of the Metamaterial}

It is convenient to realize the required negative- $\mu$ material using a resonant ring structure. The rings are fabricated on FR4 substrate via standard photolithography methods. They are then cut out and placed in a styrofoam slab placeholder which is then placed inside a microstrip waveguide for electric field measurements. Capacitively loaded rings were used to realize an effective negative permeability as proposed in [10]. The ring is essentially a series $R L C$ circuit that produces a resonant response at the frequency $\omega_{0}=(L C)^{-1 / 2}$, with $R$ accounting for the ohmic losses due to the finite conductivity of the metal. For a desired operating frequency, the inductance and capacitance of the ring can be chosen accordingly by controlling the trace width and gap spacing (seen in Fig. 4). Thicker trace widths reduce $L$, and larger gaps reduce $C$. Electromagnetically, the purpose of this ring structure is to provide the slab with an effective net negative permeability by inducing a large magnetic dipole moment. At frequencies just above resonance, the total impedance of the loop is positive imaginary, and thus the current in the loop which is proportional to $-\left(1-\omega_{0}^{2} / \omega^{2}\right)^{-1}$, creates a magnetic dipole that opposes the incident magnetic field, resulting in a permeability less than unity. For a magnetically resonant material, the relative permeability can be expressed as [10]

$$
\mu_{r}(\omega)=1+\frac{F \omega^{2}}{\omega_{0}^{2}-\omega^{2}+j \omega \Gamma}
$$

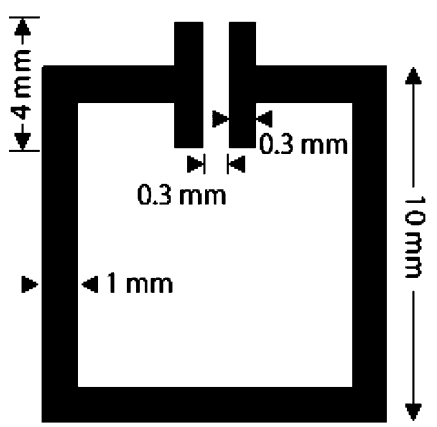

Fig. 4. Schematic of finalized ring design from Ansoft HFSS.

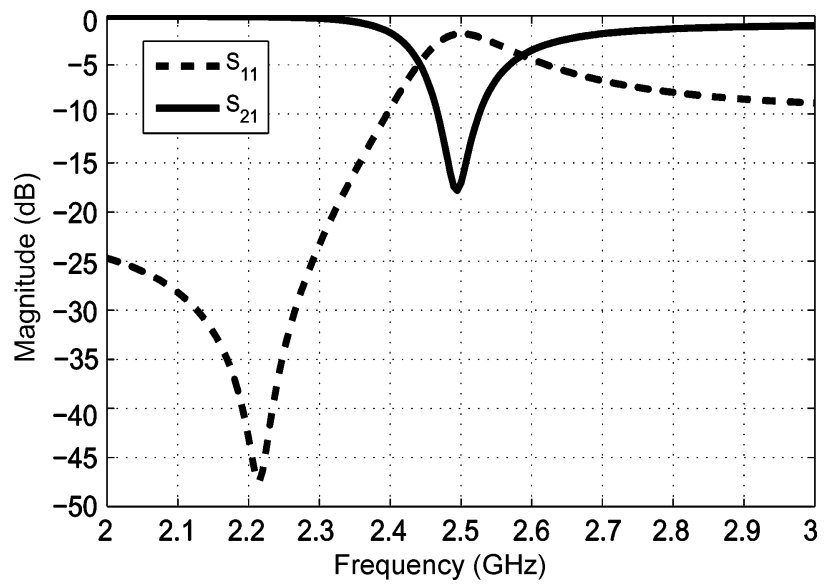

Fig. 5. Recovered $S$-parameters of the ring design from Fig. 4. The drop in $S_{21}$ at $2.5 \mathrm{GHz}$ implies that we should expect a strong resonance in $\mu_{r 2}$ because of the strong currents induced in the rings.

The form of (8) clearly shows a paramagnetic response for $\omega$ slightly less than $\omega_{0}$ and a diamagnetic response for $\omega$ slightly larger than $\omega_{0}$. When $\omega$ is right beyond $\omega_{0}, \mu_{r}$ may become negative, which is exactly what we need to create our compact cavity resonator.

This medium will be measured in a microstrip waveguide that has lowest order cutoff modes at $5 \mathrm{GHz}$. To be safely in a single TEM mode regime, the rings should be resonant between 2 and $3 \mathrm{GHz}$. In this frequency band, the free space wavelength is between $10-15 \mathrm{~cm}$, and because the unit cells should be approximately $\lambda_{0} / 8$ or smaller in thickness for an effective medium picture [10]-[12] to be appropriate, we target the unit cell size to be 1-2 cm. A parametric sweep in Ansoft HFSS was used to find a ring geometry that would resonate at $2.5 \mathrm{GHz}$ with a unit cell size of $1.5 \mathrm{~cm}$. The resulting particle and cell design is shown in Fig. 4. Fig. 5 shows the simulated $S$-parameters of a single unit cell, where the dip in $S_{21}$ at $2.5 \mathrm{GHz}$ confirms its resonance. Fig. 6 shows the effective permeability of the ring utilizing the extraction method discussed in [13]. Fig. 6 confirms that this cell design achieves a relatively low loss negative permeability in the $2.5-2.6 \mathrm{GHz}$ frequency range, as needed to realize the resonator. The extracted negative permittivity for this unit cell design was approximately 1.5.

\section{Simulations of Spatial Field Structure}

Our ultimate goal is to measure spatial field distributions like those shown in Fig. 3 using the magnetic resonant particle de- 


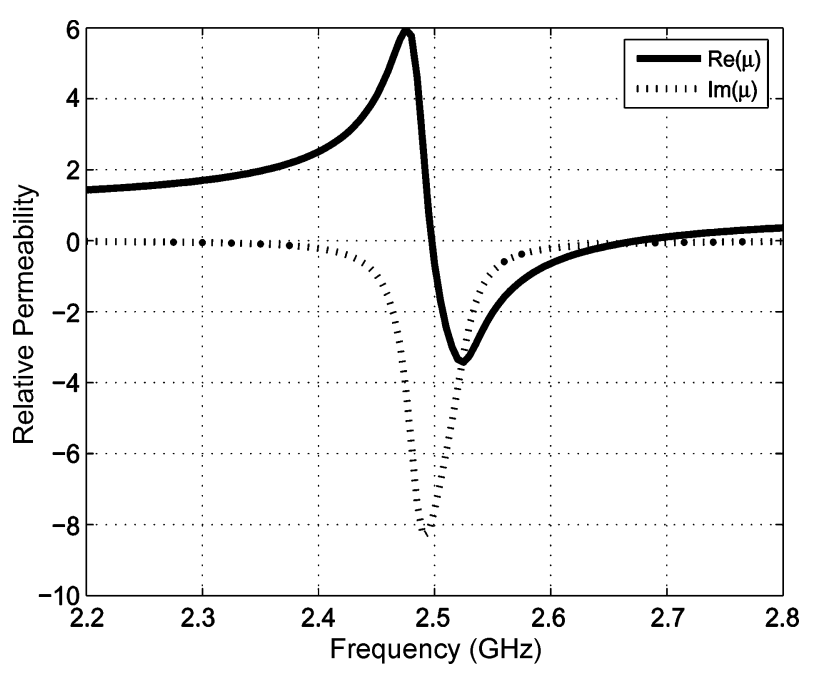

Fig. 6. Recovered permeability of the ring design from Fig. 4. We see the permeability is negative from about $2.5-2.65 \mathrm{GHz}$, implying a diamagnetic response of the ring. The extracted permittivity (not shown) $\epsilon_{r}$ was $\approx 1.5$.

signed in Section III. At issue are the conditions under which the fields in an array of macroscopic particles match those in an idealized continuous medium. This issue was explored in [14] through full wave simulations and it was found that the fields in a metamaterial can match well with effective medium fields provided that the field measurements are made as far as possible from the individual metallic inclusions. This idea has also been confirmed experimentally in [14], [15].

HFSS simulations were used to confirm that the effective medium fields can be measured in experiment for this metamaterial configuration. To simulate the TEM wave impinging upon the unit cell structure, PEC and perfect magnetic conducting (PMC) boundaries were applied on the side planes parallel to and perpendicular to the plane of the ring, respectively. Because the boundary conditions imposed will create images of the unit cell in the transverse directions, this model is really simulating a metamaterial slab of infinite extent in the transverse plane of the unit cell structure. The electric field versus position was calculated along 3 different lines in the HFSS simulation, as shown in Fig. 7. Line 1 is directly above and quite close to the copper ring, while lines 2 and 3 are along the PMC boundary and are farther from the ring. Fig. 8 shows the spatial field distributions along these 3 lines at $f=2.52 \mathrm{GHz}$, where $\mu_{r 2}<0$. Inside the metamaterial along line 1 , the fields are nothing like the effective medium fields because the quasi-static fields around the ring are much larger than the spatially averaged fields. But along lines 2 and 3, which are halfway between adjacent rings, the fields are very close to the fields predicted by effective medium theory. This suggests that the effective medium fields of this resonator structure can be essentially measured directly, provided the measurements are not made too close to the rings. In the experiment described in the next section, the fields are sampled between rings using a $7.5 \mathrm{~mm}$ long thin wire probe, which makes the measured fields effectively an average between lines 2 and 3.

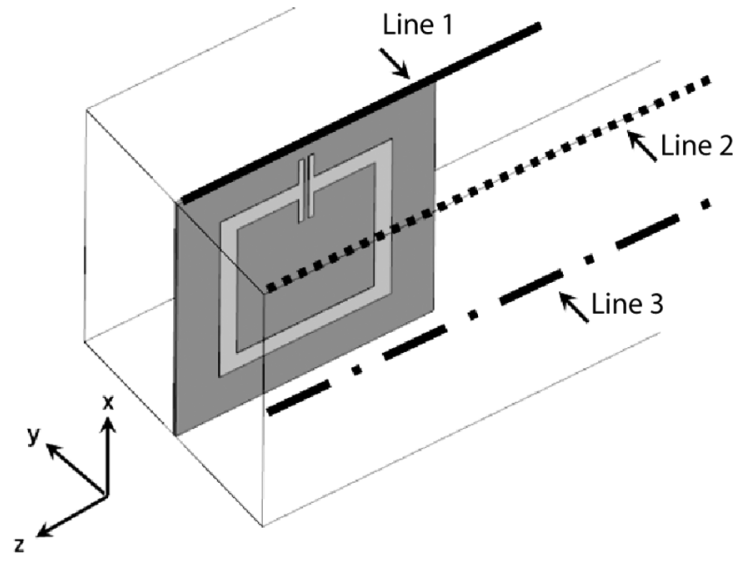

Fig. 7. HFSS model used to show that the ring structure behaves as an effective medium. The lines show where the electric field magnitude $E_{x}$ was plotted. On line 1, the strong quasi-static fields induced by the rings ruin the effective medium picture. On lines 2 and 3, which are spaced at the maximum distance from the ring yield a close match with the fields predicted by effective medium theory.

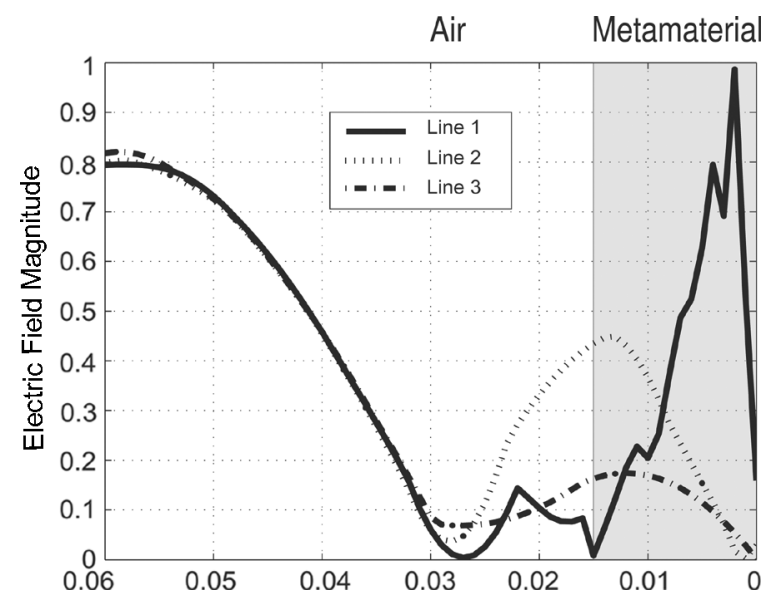

Fig. 8. Simulation results of the electric field inside the cavity when loaded with resonant rings shown in Fig. 7. Notice for line 1, the quasi-static fields due to the ring dominate the spatially averaged fields yielding poor results. Lines 2 and 3, which are farther away from the ring give a reasonable match with effective medium theory.

\section{EXPERIMENTAL RESULTS}

To verify our theoretical and simulation results, a metamaterial slab was fabricated using the ring design of Fig. 4. All field measurements were made inside a $38 \mathrm{~cm}$ long aluminum microstrip waveguide. In the center of the waveguide, the width of the upper plate is $15 \mathrm{~cm}$ and the conductor separation is $3 \mathrm{~cm}$, providing TEM operation for $f<5 \mathrm{GHz}$. This width to height ratio is kept constant as the microstrip tapers to SMA connectors at both ends, which gives a $50 \Omega$ impedance at all positions along the waveguide (Fig. 9). The upper plate contains a narrow slot through which the thin wire probe is inserted to measure the spatial field distribution.

The metamaterial slab consisted of ten unit cells in the transverse section of the waveguide, two unit cells in the vertical direction, and one unit cell in the propagation direction. Two 


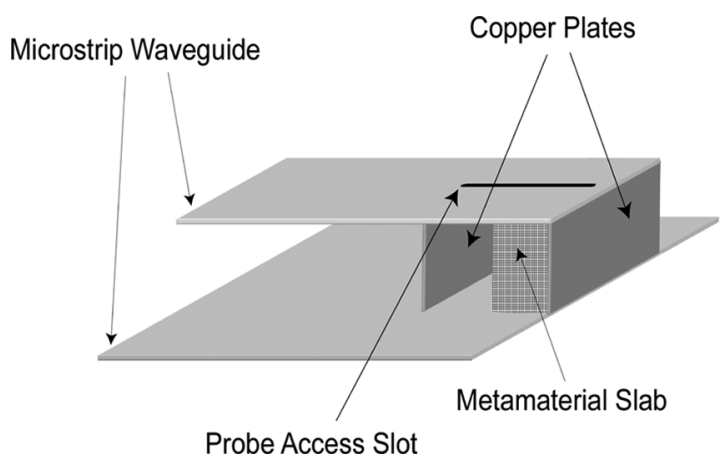

Fig. 9. Experimental setup showing the metamaterial slab loaded in the microstrip waveguide. All field measurements were made with a $7.5 \mathrm{~mm}$ wire probe that protruded through the measuring slot shown above. The waveguide was $3 \mathrm{~cm}$ in height and passes TEM modes for $f<5 \mathrm{GHz}$.

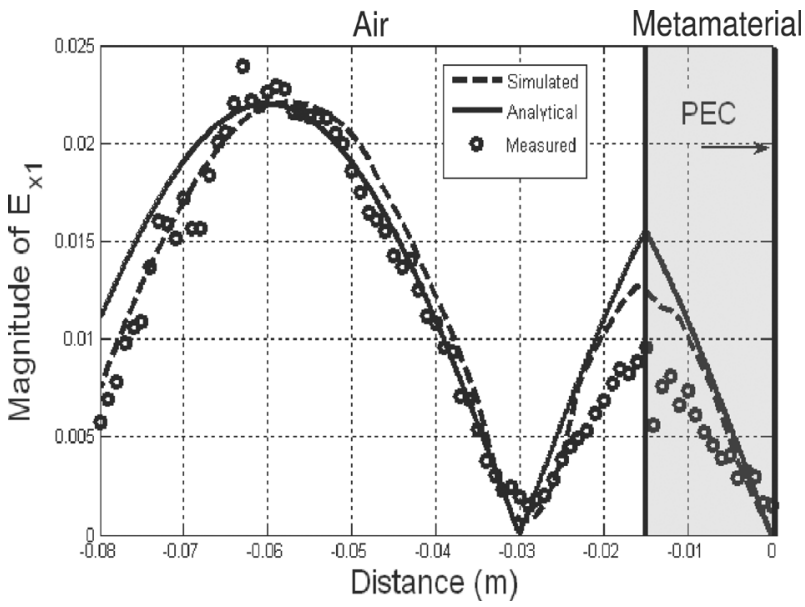

Fig. 10. Comparison plot showing the theoretical, simulated, and measured fields inside for the cavity resonator. Note that the compact cavity resonator can be formed by placing a PEC wall at $z=-0.03 \mathrm{~m}$.

rings each on FR4 slabs were slid into grooves cut in a Styrofoam wedge that fit snugly into the waveguide. A copper insert was used to approximate the needed PEC termination of the waveguide. Fig. 9 shows a sketch of this experimental setup. All field measurements were made using an HP8720A Network Analyzer.

An input signal was applied on the left side of the waveguide (Fig. 9), and the sliding probe measured the field versus position. Measurements were made every $1 \mathrm{~mm}$ throughout the waveguide, resulting in 15 measurements inside the metamaterial unit cell. Fig. 10 overlays the theoretical, the HFSS-simulated, and the measured electric field magnitudes in the cavity. The measured fields exhibit exactly the spatial variation predicted by analytical theory, namely an approximately triangular shape and a strong field null at a distance away from the air/metamaterial interface equal to the width of the negative permeability metamaterial slab. In this case, the separation between the null and the PEC plate is very close to $\lambda_{0} / 4$. These measurements clearly confirm the basic physics behind the subwavelength resonator originally described in [2]. The gradient of the electric field distribution contains essentially a near discontinuity due to the discontinuity in effective permeability, and this can produce a field null electrically close to the PEC plate. A smaller metamaterial

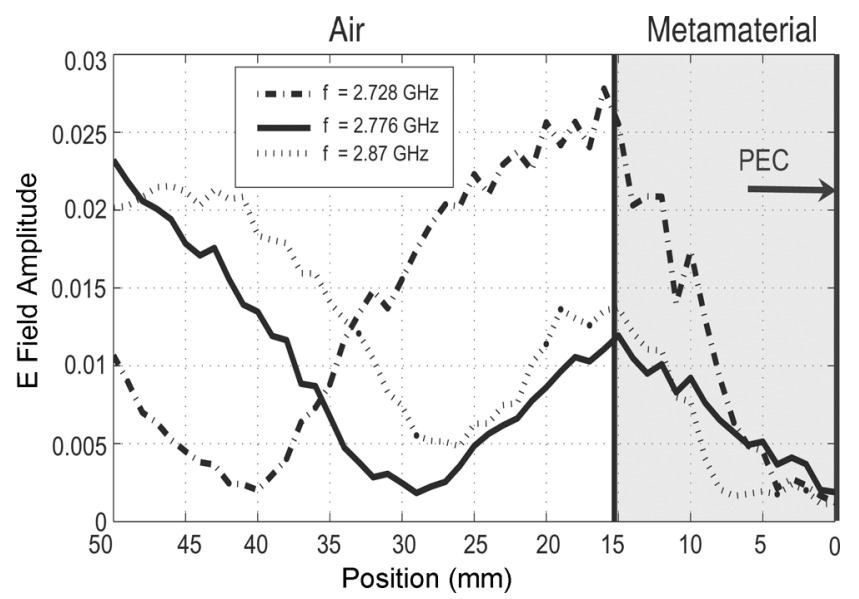

Fig. 11. Overlay of measured electric field in the cavity for frequencies near resonance. Notice as the frequency increases, the null in the electric field shifts closer to the interface, as expected theoretically since increases in frequency above the magnetic resonance make $\mu_{r 2} \rightarrow 0$, and the effective size of the cavity approaches the unit cell thickness $d$.

unit cell might have enabled an even smaller effective cavity, but designing a low loss unit cell that is significantly smaller than the $\lambda_{0} / 8$ achieved here is quite challenging.

Small losses in the fabricated metamaterial prevent the measured null from having precisely zero fields, as expected theoretically and shown in Fig. 3. This simply translates to a finite $Q$ resonance, as will be discussed soon. Although the rings were designed for $2.5 \mathrm{GHz}$, the gap size was at the limit of the resolution of the lithography process, and small changes in the gap size will cause the resonance to shift quite drastically, which is why the resonance in the fabricated rings occurred at $2.7 \mathrm{GHz}$ and not $2.5 \mathrm{GHz}$. The measured resonant frequency of the rings in the waveguide was close to $2.7 \mathrm{GHz}$, and the measured electric field in Fig. 10 is shown at a frequency of $2.776 \mathrm{GHz}$.

Fig. 11 shows the measured electric field magnitude for frequencies slightly shifted from the $2.776 \mathrm{GHz}\left(\mu_{r 2}=-1\right)$ frequency. Below this frequency, the effective permeability will be negative and larger than unity in magnitude. Equation (5) and analytical calculations in Fig. 2 show that this results in a null farther from the interface, which is precisely what was measured at $2.728 \mathrm{GHz}$. Above the $\mu_{r 2}=-1$ frequency, $\mu_{r 2}$ approaches zero and the null moves closer and closer to the interface, which again is observed experimentally at $2.86 \mathrm{GHz}$. The loss tangent $\tan \delta=\mu_{r 2}^{\prime \prime} / \mu_{r 2}^{\prime}$ become more sensitive to $\mu_{r 2}^{\prime}$ variations in this case, and becomes infinite when $\mu_{r 2}^{\prime}=0$. This explains why the measured null becomes less and less distinct as frequency increases to where $\mu_{r 2}^{\prime}$ approaches zero. In order to confirm that the fabricated metamaterial slab acts as a compact cavity resonator, the slab was sandwiched in between two copper plates, leaving an air gap equal in width to the metamaterial. $S_{11}$ versus frequency was measured using the same thin wire probe placed at the air/metamaterial interface. We also measured the same $S_{11}$ in a gap of the same width but without the metamaterial. Fig. 12 shows that both the loaded and unloaded resonators exhibit a resonance at just above $5 \mathrm{GHz}$, which corresponds to the ordinary $\lambda_{0} / 2$ resonance for a $3 \mathrm{~cm}$ plate separation. The metamaterial loaded cavity also exhibits a resonance at $2.78 \mathrm{GHz}$, 
essentially exactly the frequency for which the spatial field distribution was consistent with a resonance. This resonance is completely absent for the unloaded cavity, as expected. These measurements clearly show strong coupling to a resonant mode that is very close to predictions by the field measurements from Fig. 10 and confirm that the metamaterial slab can indeed be used to form a subwavelength cavity through the basic physics outlined in [2]. It was noted above that, ideally, the null position should move closer to the edge of the metamaterial as $\mu_{r 2}$ tends to 0 . Thus suggests that we might be able to achieve an even more compact resonator at the $\mu_{r}=0$ frequency. In this case, the effective medium picture says that the field null is located right at the interface between the air and metamaterial boundary, and the cavity effectively becomes just the metamaterial. As $\mu_{r 2}$ tends to zero, the gradient $\partial k_{2} / \partial z$ approaches zero as does the phase variation across the slab to satisfy the resonance condition. The effective medium fields for the metamaterial must vanish in this case since $\partial k_{2} / \partial z=0$ implies a trivial zero field solution in the slab.

Even though the effective medium fields tend towards zero everywhere in this case, the quasi-static fields associated with the unit cell structure itself do not. Thus a self resonant ring unit cell in a PEC box should be a resonator, although it will be one in which the quasi-static fields of an individual resonant particle dominate over the effective medium fields. That this structure can resonate is not surprising, because the metamaterial is composed of self-resonant objects. Each unit cell in air is by itself a resonator, and the effective medium picture simply says that one can put a unit cell in a PEC box and it will still resonate.

To test the possibility of the even smaller $\mu_{r 2}=0$ resonator, the copper plates were placed immediately on either side of the metamaterial slab (i.e., no air gap). The dotted line in the top panel of Fig. 12 shows the resulting $S_{11}$ versus frequency. There is a distinct resonance observed at $2.87 \mathrm{GHz}$, which is higher than the $\mu_{r 2}=-1$ frequency as expected for a $\mu_{r 2}=0$ resonance. The thin wire probe couples less strongly to this mode because the field structure of the resonance is dominated by the quasi-static fields of an individual loop, in which the electric field is primarily contained in the capacitive gap of the rings. A probe better matched to this field structure would produce a stronger resonant response. In this case, the electrical size of the resonator is smaller by roughly a factor of two (now $\lambda_{0} / 8$ ) because the cavity has been shrunk to $15 \mathrm{~mm}$. If the goal is simply to make the smallest resonator possible, this "resonant particle in a box" solution produces resonators half the size of the dual layer $\mu=-1 / \mu=1$ structure, but they appear to be quite lossy.

Fig. 12 contains two panels that show the behavior of the cavity across two frequency bands. The top panel has three plots which show $S_{11}$ for various cavity configurations. The $S_{11}$ loaded plot (solid trace) was the actual measured $S_{11}$ in the cavity when the metamaterial slab was present. In this configuration, a second copper plate was added in the microstrip waveguide to form the boundaries of a cavity that was $3 \mathrm{~cm}$ in thickness. As can be seen, the measured response of the bilayer cavity at about $2.776 \mathrm{GHz}$ tells us that we are coupling fairly strongly to a resonant mode. The $S_{11}$ unloaded plot in
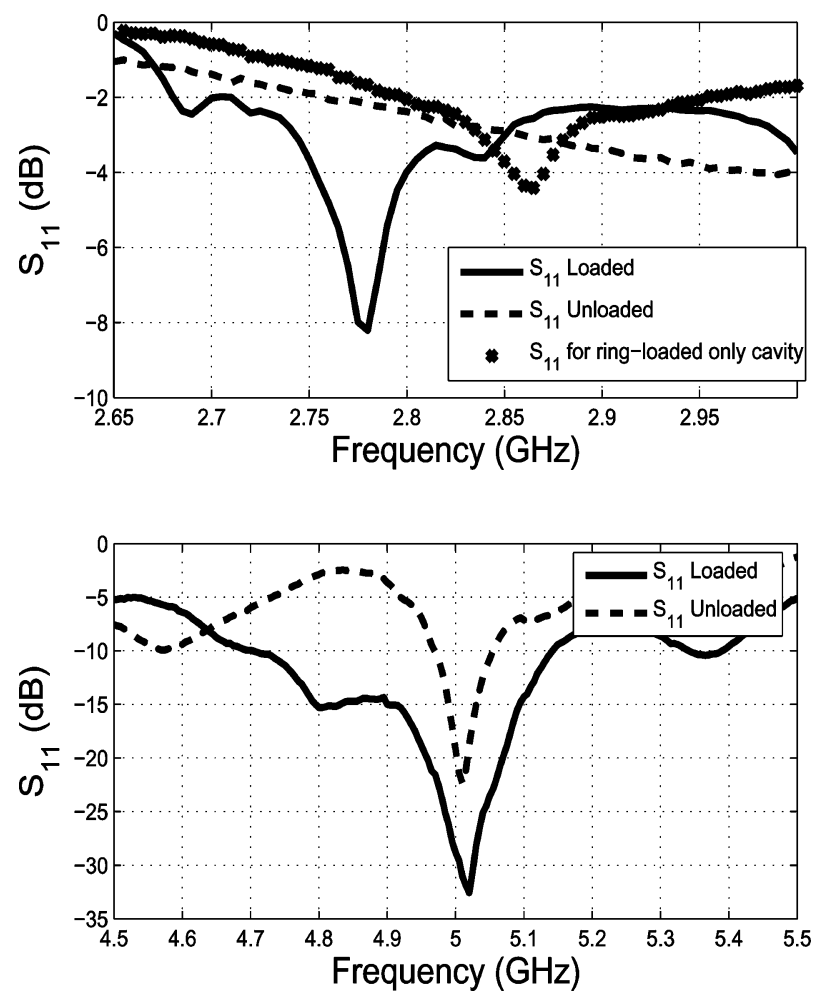

Fig. 12. Coupling to resonant modes for the loaded and unloaded cavities. It is clearly seen in the top panel that the resonance at $2.776 \mathrm{GHz}$ is only excited when the metamaterial slab is present. The dotted plot shows coupling to a resonant mode at about $2.87 \mathrm{GHz}$ when the size of the cavity is roughly the size of the slab thickness, $d$. The bottom panel clearly shows that loading the cavity with the metamaterial slab does not tamper with the $\lambda_{0} / 2$ resonance at $5 \mathrm{GHz}$. Copper plates were used to form the cavity, and the $7.5 \mathrm{~mm}$ probe was used to measure $S_{11}$ in the center of the cavity where $E_{x}$ was maximum.

this same panel (dashed trace) shows that this resonant mode is not present in the absence of the metamaterial slab. $S_{11}$ was measured in the middle of the cavity, where the maximum $E_{x}$ was expected. We deduce that the resonance at $f \approx 2.776 \mathrm{GHz}$ has a loaded quality factor $Q_{l}$ of about 173. $Q_{l}$ was determined using $S_{11}$ data, where it can be shown that $\sqrt{1 /\left|S_{11}\right|^{2}-1}$ has a Lorentzian form from which $Q_{l}$ can be extracted accurately. There were not enough data points taken around the resonant frequency of $S_{11}$ to accurately determine the unloaded quality factor $Q_{u}$, and it is not our objective to extract its precise value since the already low-loss $Q_{l}$ resonance yields a lower bound on $Q_{u}$. The actual resonator losses are smaller since $Q_{l}$ takes into account the loading effects of the probe and network analyzer.

Next, the second copper plate was pushed in as to reduce the thickness of the cavity to size $d=15 \mathrm{~mm}$, the width of the metamaterial slab. In this setup, the air layer in the cavity was absent, and the dip in $S_{11}$ at $f \approx 2.87 \mathrm{GHz}$ is the resulting response of such a structure. From the figure, it appears that the $Q$ of this resonance is smaller than the $Q$ of the $f=2.776 \mathrm{GHz}$ resonance. As the frequency is increased to where $\mu_{r 2}$ approaches zero, the loss tangent $\tan \left(\mu_{r 2}^{\prime \prime} / \mu_{r 2}^{\prime}\right)$ becomes very sensitive to variations in $\mu_{r 2}^{\prime}$, and thus our response appears to be weaker. If we look at Fig. 11, we can see that as $f$ increases to where $\mu_{r 2}$ approaches zero, it becomes harder for the $\left|E_{x 1}\right|$ to reach a null 
since $\mu_{r 2}^{\prime \prime} / \mu_{r 2}^{\prime}$ becomes large. Again, for these measurements $S_{11}$ was probed in the center of the cavity at $z=-d / 2$ where $E_{x}$ was maximum.

The bottom panel of Fig. 12 shows the response of the cavity between 4.5 and $5.5 \mathrm{GHz}$. In this measurement, the copper plates were $3 \mathrm{~cm}$ apart, and shown is $S_{11}$ when the cavity was loaded with the metamaterial slab (solid trace) as well as when the cavity was unloaded (dashed trace). The response at $5 \mathrm{GHz}$ corresponds to the $\lambda_{0} / 2$ resonance since the cavity is $3 \mathrm{~cm}$ in thickness. It is clear that the presence of the metamaterial slab does not shift this $\lambda_{0} / 2$ resonance. The $Q$ for this resonance decreases slightly upon the insertion of the slab, but this is expected and is primarily due to the ohmic losses in the copper rings since the skin depth into the copper is only a few microns.

\section{SUMMARY}

The purpose of this study is to elucidate the physics behind a metamaterial loaded resonator by measuring the spatial electric field magnitude throughout a cavity composed of an air and metamaterial bilayer. Experimental results confirm that a thin subwavelength cavity resonator can be formed using a negative- $\mu$ only metamaterial. We have shown that the field structure inside the cavity is a strong function of the metamaterial slab permeability and weakly dependent on its permittivity when the metamaterial slab is electrically thin. Measurements clarify the thin resonator concept by showing the approximately triangular electric field distribution inside the bilayer cavity. This is important, as this study visually shows the strong relationship between field structure and the metamaterial's effective permeability. The ability to reduce the size of these resonators can lead to very interesting applications in microwave circuits.

\section{REFERENCES}

[1] V. G. Veselago, "The electrodynamics of substances with simultaneously negative values of $\epsilon$ and $\mu$," Sov. Phys. Usp, p. 509, 1968.

[2] N. Engheta, "An idea for thin subwavelength cavity resonators using metamaterials with negative permittivity and permeability," IEEE Trans. Antennas Propag., vol. 1, pp. 10-12, 2002.

[3] A. E. Centeno and P. S. Excell, "High-Q dielectrically loaded electrically small cavity resonators," IEEE MGWL, vol. 3, no. 6, pp. 173-174, 1993.

[4] Z. M. Hejazi and P. S. Excell, "Miniature HTS spiral cavity resonator for mobile telephone bands," Int. J. Electron, vol. 86, pp. 117-126.

[5] J. K. Plourde and C.-L. Ren, "Application of dielectric resonators in microwave components," IEEE Trans. Microwave Theory Tech., vol. 29, pp. 745-770, 1981.

[6] Y. Li, L. Ran, H. Chen, J. Huangfu, X. Zhang, K. Chen, T. Grzegorczyk, and J. A. Kong, "Experimental realization of a one-dimensional LHM-RHM resonator," IEEE Trans. Microwave Theory Tech., vol. 53, pp. 1522-1526, 2005.

[7] S. A. Cummer and B.-I. Popa, "Wave fields measured inside a negative refractive index metamaterial," App. Phys Lett., vol. 85, no. 20, pp. 4564-4566, 2004.

[8] S. A. Tretyakov, S. I. Maslovski, I. S. Nefedov, and M. K. Krkkinen, "Evanescent modes stored in cavity resonators with backward-wave slabs," Microwave and Opt. Technol. Lett., vol. 38, no. 2, pp. 153-157, 2003.

[9] S. Hrabar, J. Bartolic, and Z. Sipus, "Experimental investigation of subwavelength resonator based on backward-wave meta-material," in Proc. IEEE Antennas and Propagation Society Int. Symp., Jun. 2004, vol. 3, pp. 2568-2571.
[10] J. B. Pendry et al., "Magnetism from conductors and enhanced nonlinear phenomena," IEEE Trans. Microwave Theory Tech, vol. 47, pp. 2075-2084, 1999.

[11] J. B. Pendry, A. J. Holden, W. J. Stewart, and I. Youngs, Phys. Rev. Lett., vol. 76, p. 4773, 1996.

[12] A. Sihvola, "Electromagnetic mixing formulae and applications," IEE Electromagn. Waves Series, vol. 47, 2000.

[13] D. R. Smith, S. Schultz, P. Markos, and C. M. Soukoulis, "Determination of effective permittivity and permeability of metamaterials from reflection and transmission coefficients," Phys. Rev. B, vol. 65, no. 195104, pp. 1-5, 2002.

[14] B.-I. Popa and S. A. Cummer, "Determining the effective electromagnetic properties of negative-refractive-index metamaterials from internal fields," Phys. Rev. B., vol. 72, no. 165102, pp. 1-5, 2005.

[15] B. J. Justice, J. J. Mock, L. Guo, A. Degiron, D. Schurig, and D. R. Smith, "Spatial mapping of the internal and external electromagnetic fields of negative index metamaterials," Opt. Exp., vol. 14, pp. 8694-8705, 2006.

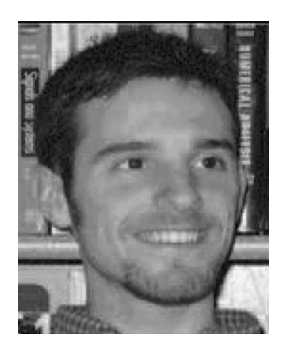

Thomas Hand (S'06) received the B.S. degree in electrical engineering and applied mathematics from the Florida Institute of Technology, Melbourne, and the M.S. degree in electrical engineering from Duke University, Durham, NC, where he is currently working toward the Ph.D. degree.

His research focus is on artificially engineered electromagnetic materials (metamaterials).

Mr. Hand was a recipient of a National Collegiate Inventors and Innovators Alliance Grant to fund his undergraduate senior design project, as well as the Charles R. Vail Outstanding Graduate Student Teaching Assistant Award in 2006.

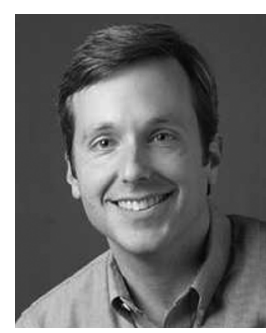

Steven A. Cummer (M'97-SM'02) received the $\mathrm{Ph} . \mathrm{D}$. degree in electrical engineering from Stanford University, Stanford, CA, in 1997.

Previously, he spent two years at NASA Goddard Space Flight Center as an NRC Postdoctoral Research Associate. He is currently an Associate Professor of electrical and computer engineering at Duke University, Durham, NC. His current work is in a variety of theoretical and experimental electromagnetic problems related to geophysical remote sensing and engineered electromagnetic materials. He has developed and implemented novel techniques for upper atmospheric remote sensing using natural electromagnetic sources, lightning remote sensing, and space plasma tomography using multi-satellite radio propagation measurements. He has written or coauthored more than 65 papers in refereed journals.

Dr. Cummer received a National Science Foundation CAREER Award and a Presidential Early Career Award for Scientists and Engineers (PECASE) in 2001.

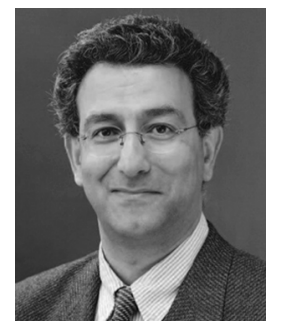

Nader Engheta (S'80-M'82-SM'89-F'96) received the B.S. degree from the University of Tehran, Iran, in 1978 and the M.S. and Ph.D. degrees from the California Institute of Technology (Caltech), in 1979 and 1982, respectively, all in electrical engineering.

After spending one year as a Postdoctoral Research Fellow at Caltech and four years as a Senior Research Scientist as Kaman Sciences Corporation's Dikewood Division in Santa Monica, CA, he joined the faculty of the University of Pennsylvania, Philadelphia, in July 1987, where he is currently the H. Nedwill Ramsey Professor of Electrical and Systems Engineering and also holds an appointment in the Bioengineering Department. He is also a member of the David Mahoney Institute of Neurological Sciences. He was the Graduate Group Chair of Electrical Engineering from July 1993 to June 1997. He is the co-editor of the book 
Metamaterials: Physics and Engineering Explorations (Wiley-IEEE Press, 2006). His current research interests and activities span over a broad range of areas including metamaterials and plasmonics, nanooptics and nanophotonics, nanocircuits and nanostructures modeling, bio-inspired/biomimetic polarization imaging and reverse engineering of polarization vision, miniaturized antennas and nanoantennas, hyperspectral sensing, biologically-based visualization and physics of sensing and display of polarization imagery, through-wall microwave imaging, millimeter-wave lensing systems, fractional operators and fractional paradigm in electrodynamics.

Dr. Engheta is a Guggenheim Fellow, a recipient of the IEEE Third Millennium Medal, and a Fellow of the Optical Society of America. He is a member of the American Physical Society (APS), the American Association for the Advancement of Science (AAAS), Sigma Xi, Commissions B, D, and K of the U.S. National Committee (USNC) of the International Union of Radio Science (URSI), and a member of the Electromagnetics Academy. He is the Vice-Chair/Chair-Elect of Commission B of USNC-URSI for 2006-2008. $\mathrm{He}$ has received various awards and distinctions for his scholarly research contributions and teaching activities including selection as one of the 2006 Scientific American 50 leaders in science and technology, the UPS Foundation Distinguished Educator Term Chair, the Fulbright Naples Chair award, and an NSF Presidential Young Investigator (PYI) award. He was twice awarded the S. Reid Warren, Jr. Award from UPenn's School of Engineering and Applied
Science, and received the Christian F. and Mary R. Lindback Foundation Award, and the W. M. Keck Foundation's 1995 Engineering Teaching Excellence Award. He is an Associate Editor of the IEEE ANTENNAS AND WIRELESS PROPAGATION LETTERS (2002-present), and was an Associate Editor for the IEEE TRANSACTIONS ON ANTENNA AND PROPAGATION (1996-2001), and Radio Science (1991-1996). He was on the Editorial Board of the Journal of Electromagnetic Waves and Applications. He served as an IEEE Antennas and Propagation Society Distinguished Lecturer for the period 1997-99. He was the Chair (1989-91) and Vice-Chair (1988-89) of the joint chapter of the IEEE ANTENNAS AND PROPAGATION/MicrowaVe THEORY AND TECHNiQueS in the Philadelphia Section. He served as a member of the Administrative Committee (AdCom) of the IEEE Society of Antennas and Propagation from January 2003 till December 2005. He has been a Guest Editor/Co-Editor several special issues, namely, the special issue of the Journal of Electromagnetic Waves and Applications on the topic of "Wave Interaction with Chiral and Complex Media" in 1992, part special issue of the Journal of the Franklin Institute on the topic of "Antennas and Microwaves" (from the 13th Annual Benjamin Franklin Symposium) in 1995, the special issue of Wave Motion on the topic of "Electrodynamics in Complex Environments" in 2001, and the IEEE TRANSACTIONS ON ANTENNAS AND PROPAGATION Special Issue on Metamaterials in 2003. 